DOI 10. 18307/2017. 0227

(C) 2017 by Journal of Lake Sciences

\title{
湖泊沉积物中磷酸盐氧同位素前处理方法对比
}

\author{
张秀梅 $^{1,2,3}$, 王亚苾 ${ }^{1,2}$, 马书占 ${ }^{1,4}$, 陈向超 ${ }^{1,2}$, 冯慕华 ${ }^{1 * *}$ \\ (1: 中国科学院南京地理与湖泊研究所湖泊与环境国家重点实验室, 南京 210008) \\ (2: 中国科学院大学, 北京 100049) \\ (3: 中国科学院大学中丹学院, 北京 100190) \\ (4: 苏州科技大学环境科学与工程学院, 苏州 215011)
}

\begin{abstract}
摘 要: 磷酸盐氧同位素 $\left(\delta^{18} \mathrm{O}_{\mathrm{P}}\right)$ 是一种有效的磷源示踪方式. 由于湖泊沉积物的组成十分复杂, 必须对样品进行除碳 处理和纯化处理. 本文对目前应用较为广泛的几种前处理方法进行比较, 包括 $\mathrm{NaClO}$ 处理和 $\mathrm{H}_{2} \mathrm{O}_{2}$ 处理等除有机碳方法, 以及 Blake 法和 McLaughlin 法等纯化方法, 以期获得适用于湖泊沉积物磷酸盐氧同位素的前处理方法. 结果表明: (1) Na$\mathrm{ClO}$ 处理可以保证较高的无机磷提取效率, 同时能有效地减少无机磷提取液中有机质含量, 且对不同形态磷的破坏较小; $\mathrm{H}_{2} \mathrm{O}_{2}$ 对有机质的去除效果不稳定, 且处理后样品磷形态之间发生转化, 显著增加了提取的无机磷浓度. (2) Blake 法和 McLaughlin 法分别采用磷钼酸铵 (APM) +磷酸铵镁 (MAP) 沉淀和 $\mathrm{CePO}_{4}$ 沉淀对样品进行纯化. 在纯化处理湖泊沉积物过 程中, Blake 法优于 McLaughlin 法, 主要体现为有机质去除率高, 并且磷的回收率较为稳定. (3) 经 Blake 法纯化丹麦 Nordborg 湖沉积物样品得到的 $\mathrm{Ag}_{3} \mathrm{PO}_{4}$ 中 $\mathrm{C} 、 \mathrm{~N}$ 含量低于 McLaughlin 法且重现性好. 本文结合 NaClO 法与 Blake 法的优点, 建立 了一种适合湖泊沉积物的磷酸盐氧同位素前处理方法: 首先用 $2.5 \% \mathrm{NaClO}$ 对沉积物样品进行除碳预处理, 然后对磷酸盐 提取液依次通过氢氧化镁共沉淀 (MAGIC)、APM+MAP 沉淀、阳离子交换树脂处理,最后生成 $\mathrm{Ag}_{3} \mathrm{PO}_{4}$ 沉淀.
\end{abstract}

关键词: 沉积物; 磷酸盐氧同位素; 前处理; 除有机碳;无机磷

\section{Comparison of pretreatment methods of oxygen isotope composition of phosphate in lake sediments}

\author{
Zhang Xiumei ${ }^{1,2,3}$, Wang Yarui $^{1,2}$, Ma Shuzhan ${ }^{1,4}$, Chen Xiangchao ${ }^{1,2}$ \& Feng Muhua ${ }^{1 * *}$ \\ (1: State Key Laboratory of Lake Science and Environment, Nanjing Institute of Geography and Limnology, Chinese Academy of \\ Sciences, Nanjing 210008, P.R.China) \\ (2: University of Chinese Academy of Sciences, Beijing 100049,P.R.China) \\ (3: Sino-Danish Center for Education and Research, Beijing 100190, P.R.China) \\ (4: School of Environmental Science and Engineering, Suzhou University of Science and Technology, Suzhou 215011, P.R.China)
}

Abstract: Oxygen isotope of phosphate $\left(\delta^{18} \mathrm{O}_{\mathrm{P}}\right)$ was a very effective proxy for tracing sources. Due to the complicated composition of lake sediments, samples must be treated by carbon elimination and purified before the $\delta^{18} \mathrm{O}_{\mathrm{P}}$ measurement. In order to establish a suitable pretreatment method for $\delta^{18} \mathrm{O}_{\mathrm{P}}$ of lake sediments, this study compared several widely-used pretreatment methods, including organic carbon elimination by $\mathrm{NaClO}$ treatment and $\mathrm{H}_{2} \mathrm{O}_{2}$ treatment, purification by Blake method and McLaughlin method. The results showed: (1) NaClO kept high inorganic phosphate extract ratios, and removed organic matter efficiently with less impacts on different phosphate species. The organic removal efficiency by $\mathrm{H}_{2} \mathrm{O}_{2}$ was not stable while the phosphate species changed after treatments and inorganic phosphate concentration increased significantly. (2) Ammonium phosphomolybdate( APM) + ammonium phosphate (MAP) and $\mathrm{CePO}_{4}$ was used by Blake method and McLaughlin method, respectively. Blake method had better performance on the purification of lake sediments samples than McLaughlin method, and a higher organic matter removal rate than McLaughlin

* 国家自然科学基金项目 $(41471075,41171366)$ 资助.2016-04-19 收稿;2016-08-11 收修改稿. 张秀梅( 1989 ), 女, 硕士研究生;E-mail: zhxm_66@yeah.net.

** 通信作者;E-mail: mhfeng@ niglas.ac.cn. 
method with stable phosphate throughput. (3) $\mathrm{The}_{\mathrm{Ag}_{3}} \mathrm{PO}_{4}$ produced by the Blake method had lower $\mathrm{C}, \mathrm{N}$ contents and better repeatability than the McLaughlin method. The advantages of both the $\mathrm{NaClO}$ treatment and the Blake method were made full use to establish an improved $\delta^{18} \mathrm{O}_{\mathrm{P}}$ pretreatment method for lake sediments with modified procedures. It is recommended to use $2.5 \% \mathrm{Na}-$ $\mathrm{ClO}$ to remove organic matter before phosphate extraction, and then purify the phosphate extractions through MAGIC, APM+MAP, and cation resin treatment, in turn.

Keywords: Sediments; oxygen isotope of phosphate; pretreatment method; organic carbon elimination; inorganic phosphate

磷循环属于典型的沉积型循环 ${ }^{[1]}$. 沉积物作为湖泊磷的蓄积库, 汇集了各种类型的陆源磷, 但在一定条 件下, 沉积物又由磷的 “汇” 转变为 “源”, 不断地释放到上覆水中 ${ }^{[2-3]}$. 因此, 探究沉积物中磷的来源, 对控制 和治理湖泊富营养化具有重要的指导意义.

目前发现的磷同位素有 23 种 $\left({ }^{24} \mathrm{P} \sim{ }^{46} \mathrm{P}\right)$, 但只有 ${ }^{31} \mathrm{P}$ 是稳定同位素, 其余均为放射性同位素,含量低、半 衰期短, 限制了它们在磷源示踪和磷循环方面的应用 ${ }^{[4]}$. 在自然界中, 磷主要以磷酸盐的形式存在, 且 $\mathrm{PO}_{4}^{3-}$ 中的 $\mathrm{P}$ 与 $\mathrm{O}$ 的结合比较稳定 (键能为 $359.8 \mathrm{~kJ} / \mathrm{mol}$ ); 在地表温度和 $\mathrm{pH}$ 范围内, 若没有生物的作用 $\mathrm{P}-\mathrm{O}$ 键不 易断裂. 基于此, 磷酸盐的氧同位素 $\left(\delta^{18} \mathrm{O}_{\mathrm{P}}\right)$ 作为一种示踪剂广泛地应用于磷的生物地球化学循环研究 ${ }^{[5-8]}$.

通常, 样品中的 $\mathrm{PO}_{4}^{3-}$ 要转化成 $\mathrm{Ag}_{3} \mathrm{PO}_{4}$, 然后采用同位素比值质谱法 (IRMS) 进行磷酸盐氧同位素测定. 然而自然界中的样品成分复杂, 尤其是有机质的存在,既影响 $\mathrm{Ag}_{3} \mathrm{PO}_{4}$ 生成, 又干扰 $\delta^{18} \mathrm{O}_{\mathrm{p}}$ 测定, 因此测定之前 需要对样品进行提取和纯化处理. 首先, 提取 $\mathrm{PO}_{4}^{3-}$ 之前对样品进行除碳处理, 可以有效地提高后续纯化效 果. Stephan 和 $O^{\prime}$ Neil 等针对有机质含量较高的生物性磷酸盐样品 (如动物骨骼、牙齿等), 分别提出了用 $2.5 \%$ $\mathrm{NaClO}+\mathrm{NaOH}$ 和 $30 \% \mathrm{H}_{2} \mathrm{O}_{2}$ 进行预处理的方法 ${ }^{[9-10]}$. $\mathrm{NaClO}$ 处理后 $\mathrm{Ag}_{3} \mathrm{PO}_{4}$ 产物颜色呈亮黄绿色, 且结晶较 大. $\mathrm{H}_{2} \mathrm{O}_{2}$ 处理可以去除有机碳,缩短了磷酸盐的提取时间,并且可生成较纯净的 $\mathrm{Ag}_{3} \mathrm{PO}_{4}$ 产物. 其次, 前处理 中最为关键的步骤是纯化提取的 $\mathrm{PO}_{4}^{3-}$, Blake 教授和 McLaughlin 教授分别提出了不同的纯化方法 ${ }^{[11-12]}$. Blake 方法首先将矿石提取液稀释至海水浓度, 用 2 次氢氧化美共沉淀 (magnesium-induced co-precipitation, 简称 MAGIC) 进行富集, 同时去除部分杂质; 接着用 $\mathrm{PO}_{4}^{3-}$ 的特征沉淀磷锄酸铵 ( APM) 和磷酸铵镁 (MAP) 进 一步纯化 $\mathrm{PO}_{4}^{3-}$; 然后依次用阳离子交换树脂除去金属离子、阴离子交换树脂除去有机质、阳离子交换树脂除 去 $\mathrm{HCO}_{3}^{-}$. McLaughlin 方法是用一次 MAGIC 富集海水中的溶解态磷酸盐, 然后用 $\mathrm{PO}_{4}^{3-}$ 的特征沉淀 $\mathrm{CePO}_{4}$ 进 一步纯化 $\mathrm{PO}_{4}^{3-}$, 再用阳离子交换树脂除去 $\mathrm{Ce}^{3+}$, 最后将 $\mathrm{PO}_{4}^{3-}$ 转化成 $\mathrm{Ag}_{3} \mathrm{PO}_{4}$. 卢阳阳等 ${ }^{[13]}$ 改进了 McLaughlin 法, 在除去 $\mathrm{Ce}^{3+}$ 后用阴离子交换树脂除有机质. Blake 法的优点是 APM 和 MAP 沉淀对 $\mathrm{pH}$ 要求较低, 容易生 成, 且沉淀量小, 对有机质的吸附作用小; 缺点是操作繁琐、耗时. McLaughlin 法的优点是 $\mathrm{CePO}_{4}$ 对 $\mathrm{Cl}^{-}$等杂质 离子有较好的去除效果; 缺点是 $\mathrm{CePO}_{4}$ 受 $\mathrm{pH}$ 影响较大, $\mathrm{Fe} 、 \mathrm{Al} 、 \mathrm{Ca}$ 等金属离子会干扰沉淀的生成. 已有的预 处理方法主要是针对海水、矿石、土壤、动物骨骼、牙齿等, 而对湖泊沉积物的研究相对较少 ${ }^{[6,9-10,12,14-15]}$. 湖泊 沉积物组成相对其余样品更加复杂, 含有大量腐殖质、蛋白质、糖、脂质等非磷酸根的含氧杂质, 因此建立一 种适用于湖泊沉积物的磷酸盐氧同位素前处理方法尤为重要.

为了探明 $\mathrm{NaClO}$ 处理和 $\mathrm{H}_{2} \mathrm{O}_{2}$ 处理等除有机碳方法, Blake 法和 McLaughlin 法等纯化方法在湖泊沉积物 磷酸盐氧同位素前处理中的效果, 本文对这几种方法进行了对比研究. 首先,采用 $2.5 \% \mathrm{NaClO}$ 和 $30 \% \mathrm{H}_{2} \mathrm{O}_{2}$ 对有机质含量较高的湖泊沉积物样品进行预处理, 并比较 2 种预处理对无机磷提取效率、提取液中溶解性 有机碳 (DOC) 含量以及沉积物磷形态的影响. 其次, 运用 Blake 法和 McLaughlin 法对沉积物提取液中磷酸 盐进行纯化, 比较 2 种方法中 DOC 的去除效率和磷酸盐的回收率. 本文结合预处理除碳方法和 2 种纯化方 法的优点, 并对一些操作步骤进行篮选优化, 建立一种适用于湖泊沉积物磷酸盐氧同位素的前处理方法.

\section{1 材料与方法}

\section{1 试剂与仪器}

试剂: $\mathrm{NaClO}$ (Aladdin)、 $\mathrm{H}_{2} \mathrm{O}_{2}$ ( AR )、 $\mathrm{HCl}(\mathrm{GR}) 、 \mathrm{Mg}\left(\mathrm{NO}_{3}\right)_{2} \cdot 6 \mathrm{H}_{2} \mathrm{O}(\mathrm{AR}) 、 \mathrm{NaOH}(\mathrm{GR}) 、 \mathrm{HNO}_{3}(\mathrm{GR}) 、$ $\left(\mathrm{NH}_{4}\right){ }_{6} \mathrm{Mo}_{7} \mathrm{O}_{24} \cdot 4 \mathrm{H}_{2} \mathrm{O}(\mathrm{AR}) 、 \mathrm{CeNO}_{3}$ (Aladdin)、阳离子交换树脂( BioRad AG-50X8, $\mathrm{H}^{+}$型, 100 200 目)、阴离 子交换树脂( BioRad AG1-X8, $\mathrm{OH}^{-}$型, 200 400 目)、 $\mathrm{NaHCO}_{3}$ (Aladdin)、 $\mathrm{MgCl}_{2}(\mathrm{AR}) 、 \mathrm{BD}$ 试剂、 $\mathrm{CH}_{3} \mathrm{COOK}$. 
$3 \mathrm{H}_{2} \mathrm{O}(\mathrm{GR}) 、 \mathrm{CH}_{3} \mathrm{COOH}(\mathrm{AR})$.

器皿及仪器: 聚四氟乙烯 (PTFE) 离心管、100 ml 烧杯、1 L 烧杯、10 ml 玻璃离心管、恒温振荡器 ( HZ$9511 \mathrm{~K}$ )、冷冻干燥机( 7948030,Labconco)、离心机( TGL-16M, 湘仪)、马弗炉 ( KBF1700)、带砂芯的层析柱、 总有机碳分析仪(Torch,Teledyne Tekmar)、元素分析仪(EA3000,EuroVector).

\section{2 样品采集}

丹麦富营养化湖泊 Nordborg 湖平均水深 $5 \mathrm{~m}$, 湖面面积 $0.546 \mathrm{~km}^{2}$,换水周期为 $0.82 \mathrm{a}^{[16]}$. 流域面积 $63 \%$ 为农田, $25 \%$ 为城镇用地, 其他区域被森林覆盖. 湖北岸设有两个前置库, 拦截净化农田来水. 于 2014 年 3 月 12 日用彼得森采泥器进行表层沉积物样品采集. 4 个样品分别位于 Nordborg 湖最深处 $(8.5 \mathrm{~m}) 、 1 / 2$ 水深处 $(4.2 \mathrm{~m})$ 、前置库 KS1 和前置库 KS2, 记为样品 1 、样品 2 、样品 3 和样品 4.4 个沉积物样品的碳、氮、磷含量如 表 1 所示.

表 1 丹麦 Nordborg 湖沉积物样品的碳、氮、磷含量

Tab.1 The contents of C, N, P of four samples in Nordborg Lake

\begin{tabular}{cccccrccc}
\hline 样品 & 纬度 & 经度 & 含水率 & $\begin{array}{c}\mathrm{TC} / \\
(\mathrm{mg} / \mathrm{g})\end{array}$ & $\begin{array}{c}\mathrm{TOC} / \\
(\mathrm{mg} / \mathrm{g})\end{array}$ & $\begin{array}{r}\mathrm{TN} / \\
(\mathrm{mg} / \mathrm{g})\end{array}$ & $\begin{array}{c}\mathrm{TP} / \\
(\mathrm{mg} / \mathrm{g})\end{array}$ & $\begin{array}{c}\mathrm{IP} / \\
(\mathrm{mg} / \mathrm{g})\end{array}$ \\
\hline 1 & $55^{\circ} 03^{\prime} 33.88^{\prime \prime} \mathrm{N}$ & $9^{\circ} 45^{\prime} 15.68^{\prime \prime} \mathrm{E}$ & $87.30 \%$ & 139.01 & 101.14 & 10.62 & 1.76 & 1.04 \\
2 & $55^{\circ} 03^{\prime} 04.50^{\prime \prime} \mathrm{N}$ & $9^{\circ} 46^{\prime} 48.51^{\prime \prime} \mathrm{E}$ & $80.16 \%$ & 122.52 & 64.19 & 7.79 & 1.20 & 0.85 \\
3 & $55^{\circ} 03^{\prime} 45.55^{\prime \prime} \mathrm{N}$ & $9^{\circ} 44^{\prime} 59.62^{\prime \prime} \mathrm{E}$ & $73.00 \%$ & 90.64 & 77.42 & 7.03 & 1.56 & 1.42 \\
4 & $55^{\circ} 03^{\prime} 45.18^{\prime \prime} \mathrm{N}$ & $9^{\circ} 47^{\prime} 37.13^{\prime \prime} \mathrm{E}$ & $69.25 \%$ & 71.12 & 49.82 & 3.77 & 1.61 & 1.30 \\
\hline
\end{tabular}

\section{3 实验方法}

1.3.1 预处理方法 将沉积物样品冻干后研磨过 200 目篮. 分别取 $0.5 \mathrm{~g}$ 左右样品于 PTFE 离心管中, 设置 3 个实验组: 对照组、 $\mathrm{NaClO}$ 处理组和 $\mathrm{H}_{2} \mathrm{O}_{2}$ 处理组. 每组处理 3 个平行. 具体步骤如下:

对照组:不进行任何预处理.

$\mathrm{NaClO}$ 处理组: 加人 $10 \mathrm{ml} 2.5 \% \mathrm{NaClO}$ 溶液震荡 $24 \mathrm{~h}$ 后离心 $(7000$ 转 $/ \mathrm{min}, 10 \mathrm{~min}$ ), 弃去上清液, 剩余 残渣用纯水洗涤 4 5 次至中性.

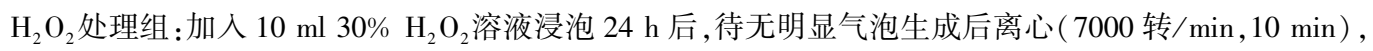
弃去上清液,剩余残渣用纯水洗涤 4 5 次至中性.

选取部分预处理后样品残渣, 用 $1 \mathrm{~mol} / \mathrm{L} \mathrm{HCl}$ 提取无机磷, 其余样品残渣按照连续萃取法 (SEDEX) 提取 5 种形态磷( 即弱交换态磷 Ex-P、铁铝结合态磷 Fe-P、自生磷灰石 CFAP、碎屑磷灰石 CAP、闭蓄态磷 Org-P $)^{[17]}$. 按照钼锑抗分光光度法测定各提取液中 $\mathrm{PO}_{4}^{3-}-\mathrm{P}$ 含量 ${ }^{[18]}$, 并且用总有机碳分析仪测定无机磷提 取液中的 DOC 浓度.

1.3.2 纯化方法 以样品 3 为例, 分别按照 Blake 法和 McLaughlin 法对沉积物提取液进行纯化处理, 并测定 每一步的 DOC 浓度和磷含量, 最后测定产物 $\mathrm{Ag}_{3} \mathrm{PO}_{4}$ 的 $\mathrm{C} 、 \mathrm{~N}$ 含量. 每组处理 7 个平行.

Blake 方法 ${ }^{[19]}$ : 依次经过 2 次氢氧化镁共沉淀 (MAGIC)、磷钼酸铵沉淀、磷酸铵镁沉淀、阳离子交换树 脂吸附金属离子、阴离子交换树脂除去有机质、阳离子交换树脂除 $\mathrm{HCO}_{3}^{-}$、微沉淀生成 $\mathrm{Ag}_{3} \mathrm{PO}_{4}, 60^{\circ} \mathrm{C}$ 烘干 待测.

McLaughlin 方法: 参照 McLaughlin 方法 ${ }^{[12]}$, 并结合卢阳阳等的方法进行改进 ${ }^{[13]}$. 依次经过阳离子交换 树脂除去 $\mathrm{Fe} 、 \mathrm{Al} 、 \mathrm{Ca}$ 等金属离子、1 次 MAGIC 操作、CePO 沉淀、阳离子交换树脂除去 $\mathrm{Ce}^{3+}$ 、阴离子交换树脂 除去有机质、加酸除去 $\mathrm{HCO}_{3}^{-}$, 快速沉淀生成 $\mathrm{Ag}_{3} \mathrm{PO}_{4}, 60^{\circ} \mathrm{C}$ 烘干待测.

\section{4 数据统计与分析}

采用 Origin 9.0 软件进行图形绘制,并用 SPSS 20.0 软件对数据进行统计分析. 


\section{2 结果与讨论}

\section{1 不同预处理方法的比较}

2.1.1 不同预处理方法对 DOC 去除效果的影响 经 NaClO 处理后无机磷提取液中 DOC 浓度减少了 $32 \%$ $74 \% ; \mathrm{H}_{2} \mathrm{O}_{2}$ 对 DOC 的去除效果不稳定, 经 $\mathrm{H}_{2} \mathrm{O}_{2}$ 处理后样品 1 和样品 4 的无机磷提取液中 DOC 浓度分别减 少了 $10 \%$ 和 $55 \%$, 但是样品 2 和样品 3 的 DOC 浓度却分别增加了 $21 \%$ 和 70\%. 对比 2 种预处理方法对样品 DOC 的去除结果发现, 两者差异显著 $(P=0.01)$, 且 $\mathrm{NaClO}$ 处理对 DOC 去除效果优于 $\mathrm{H}_{2} \mathrm{O}_{2}$ (图 1a). 这与土 壤方面的相关研究结果一致, 在去除土壤有机质时 $\mathrm{NaClO}$ 比 $\mathrm{H}_{2} \mathrm{O}_{2}$ 更有效 ${ }^{[20-23]}$.

$\mathrm{NaClO}$ 去除天然有机质时, $\mathrm{ClO}^{-}$直接与腐殖酸、富里酸、芳香族化合物等发生氯化反应,生成 $\mathrm{CHCl}_{3}$ 、氯 代乙酸等 ${ }^{[24-26]}$. 而且 $\mathrm{NaClO}$ 溶液呈碱性, 可将部分腐殖酸和富里酸洗脱出来, 从而减少无机磷提取液中 DOC 浓度.

$\mathrm{H}_{2} \mathrm{O}_{2}$ 稳定性较差, 可被沉积物中的 $\mathrm{Fe}^{3+}$ 和锰氧化物催化分解 ${ }^{[27]}$, 从而减弱对有机质的降解作用 ${ }^{[28]}$. 腐 殖质、木质素以及一些简单的碳水化合物与 $\mathrm{H}_{2} \mathrm{O}_{2}$ 反应会生成大量小分子有机酸( 如蚁酸、乙酸、乙二酸、丙 二酸)、苯酚、苯甲酸 ${ }^{[29]}$. 样品 2 和样品 3 的无机磷提取液中 DOC 浓度升高可能是因为 $\mathrm{H}_{2} \mathrm{O}_{2}$ 将沉积物样品 中部分难降解有机质转化成水溶性有机酸.

2.1.2 不同预处理方法对沉积物磷形态的影响 $\mathrm{NaClO}$ 对沉积物磷形态的影响小于 $\mathrm{H}_{2} \mathrm{O}_{2}$. 经 $\mathrm{NaClO}$ 处理后, 无机磷提取率维持在 93\% 98\% (图 1b), 但沉积物的总磷减少 14\% 29\% (图 2), 表明 NaClO 对有机磷有明 显的去除效果而对无机磷几乎无影响. 经 $\mathrm{H}_{2} \mathrm{O}_{2}$ 处理后, 除样品 1 总磷减少 $30 \%$ 外, 其他 3 种样品的总磷与对 照组几乎没有差异 (图 2), 但无机磷的提取率提高了 $35 \%$ 64\% ( 图 1b), 表明 $\mathrm{H}_{2} \mathrm{O}_{2}$ 将有机磷转化为无机磷.

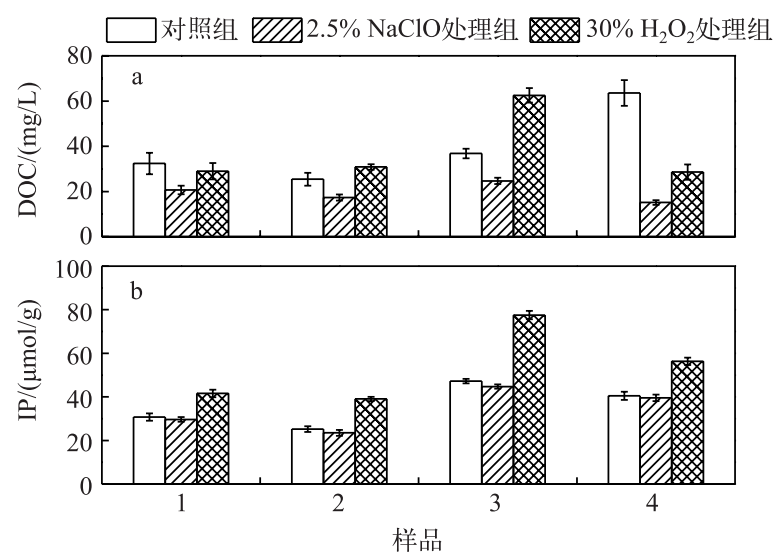

图 $12.5 \% \mathrm{NaClO}$ 和 $30 \% \mathrm{H}_{2} \mathrm{O}_{2}$ 预处理后沉积物提取液中 DOC 浓度 $(\mathrm{a})$ 和无机磷含量 $(\mathrm{b})$ 的变化

Fig. 1 Variation of DOC concentration(a) and inorganic phosphate content(b) in sediments extracts after $2.5 \% \mathrm{NaClO}$ and $30 \% \mathrm{H}_{2} \mathrm{O}_{2}$ pretreatments

比较 $\mathrm{NaClO}$ 和 $\mathrm{H}_{2} \mathrm{O}_{2}$ 处理后沉积物磷赋存形态的变化可得, $\mathrm{NaClO}$ 和 $\mathrm{H}_{2} \mathrm{O}_{2}$ 处理组的 Ex-P 分别减少了 $29 \% \sim 86 \%$ 和 86\% 90\%, 说明 Ex-P 更易受 $\mathrm{H}_{2} \mathrm{O}_{2}$ 破坏 $(P=0.039)$. Ex-P 一般通过物理吸附作用或者磷酸盐 与沉积相胶体、矿物通过配位交换的方式吸附在沉积物表面 ${ }^{[30]}$, 极易因环境的改变而释放. 实验过程中, $\mathrm{NaClO}$ 与沉积物反应缓和, 而 $\mathrm{H}_{2} \mathrm{O}_{2}$ 与沉积物反应时却有大量气泡冒出, 激烈的气泡涌动可能促进了 Ex-P 的 释放. 但由于 Ex-P 仅占总磷的 1\% 3\%, 因此 Ex-P 的变化对无机磷提取率的影响可忽略.

除样品 4 外, $\mathrm{NaClO}$ 处理组和 $\mathrm{H}_{2} \mathrm{O}_{2}$ 处理组的 $\mathrm{Fe}-\mathrm{P}$ 含量变化几乎无差异 $(P=0.666)$, 分别减少了 $43 \% \sim$ $61 \%$ 和 $37 \% \sim 65 \%$. Fe-P 是指铁铝氧化物表面及氧化物晶格包裹的磷, 包括无定型氧化铁 ( $\mathrm{FeOOH}$ ) - $\mathrm{P}$ 胶体. $\mathrm{FeOOH}-\mathrm{P}$ 最稳定的 $\mathrm{pH}$ 范围为 $6.5 \sim 8.0$, 在高 $\mathrm{pH}$ 或低 $\mathrm{pH}$ 条件下, 都易将 $\mathrm{PO}_{4}^{3-}$ 释放出来 ${ }^{[31]} . \mathrm{NaClO}$ 水解产生 $\mathrm{OH}^{-}$, 可促进 $\mathrm{Fe}-\mathrm{P}$ 的释放. 考虑到 $\mathrm{pH}$ 值较高的 $\mathrm{NaClO}$ 溶液对含铝氧化物的溶解作用, Siregar 等 ${ }^{[22]}$ 建议将 
$\mathrm{pH}$ 下调至 8 左右以减少对晶体矿物结构的破坏. $30 \% \mathrm{H}_{2} \mathrm{O}_{2}$ 呈弱酸性 ( $\mathrm{pH}$ 为 $5 \sim 6$ ), 对 $\mathrm{FeOOH}-\mathrm{P}$ 有一定的溶 解作用, 使 Fe-P 转化成其他形态的磷. $\mathrm{H}_{2} \mathrm{O}_{2}$ 处理组中样品 4 的 Fe-P 含量升高可能是因为其他形态的磷转 化成了 $\mathrm{Fe}-\mathrm{P}$, 且此转化量大于 $\mathrm{H}_{2} \mathrm{O}_{2}$ 对 $\mathrm{FeOOH}-\mathrm{P}$ 的溶解量.

$\mathrm{NaClO}$ 对 CFAP 和 CAP 影响不大, 含量波动范围分别为 - 9\% 6\% 和-4\% 3\%. CFAP 和 CAP 主要是 $\mathrm{CaCO}_{3}$ 结合态的磷, 稳定性较强, 不易释放 ${ }^{[3]} \cdot \mathrm{H}_{2} \mathrm{O}_{2}$ 使这 2 种磷形态的磷含量明显增加, 其中 CFAP 增加了 $21 \% \sim 65 \%$; CAP 除样品 3 变化较小, 仅减少了 $4 \%$ 外, 其它样品增加了 $32 \% \sim 182 \%$, 其原因可能是 $\mathrm{H}_{2} \mathrm{O}_{2}$ 在氧 化有机质的过程中将有机磷转化为无机磷.

与对照组相比, 经过 $\mathrm{NaClO}$ 和 $\mathrm{H}_{2} \mathrm{O}_{2}$ 处理后 Org-P 含量分别减少了 $47 \% \sim 69 \%$ 和 $82 \% \sim 96 \%$. 这表明 $\mathrm{NaClO}$ 和 $\mathrm{H}_{2} \mathrm{O}_{2}$ 均对 Org-P 有明显影响, 且 $\mathrm{H}_{2} \mathrm{O}_{2}$ 处理对 Org-P 影响更明显 $(P=0.020)$.

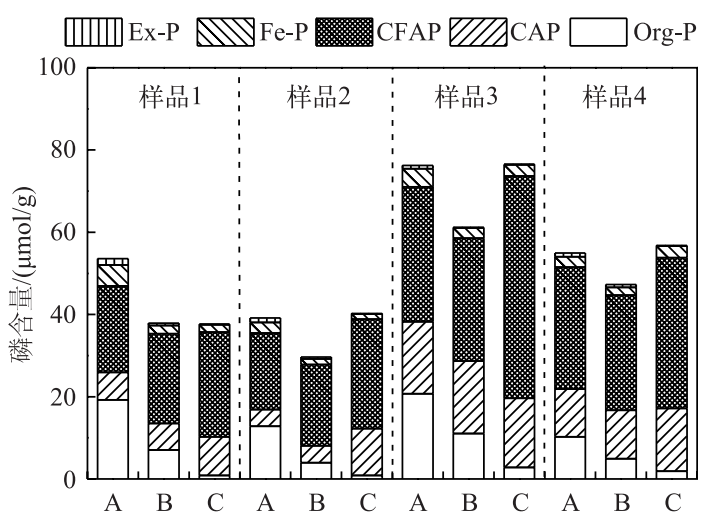

A: 无处理 B: $2.5 \% \mathrm{NaClO}$ 处理 C: $30 \% \mathrm{H}_{2} \mathrm{O}_{2}$ 处理

图 2 2.5\% $\mathrm{NaClO}$ 和 $30 \% \mathrm{H}_{2} \mathrm{O}_{2}$ 预处理后沉积物磷形态的变化

Fig.2 Variations of sediments phosphates species after 2.5\% $\mathrm{NaClO}$ and $30 \% \mathrm{H}_{2} \mathrm{O}_{2}$ pretreatments

\section{2 两种经典方法的纯化效率对比}

2.2.1 有机质去除率 比较 Blake 法和 McLaughlin 法对 $\mathrm{PO}_{4}^{3-}$ 提取液的纯化效果可得, 两种方法均使有机质含 量降低, 但是趋势不同 (图 3). 按照 Blake 法处理后 DOC 浓度呈逐步降低的趋势, 而 McLaughlin 法中 DOC 浓度却波动较大。

Blake 法和 McLaughlin 法分别采用 2 次 MAGIC 和 1 次 MAGIC 操作, DOC 浓度分别减少了 $42.03 \%$ 和 $33.98 \%$, 二者相差 $8.04 \%$, 表明第 2 次 MAGIC 操作对 DOC 的去除作用有限. 这可能与 $\mathrm{Mg}(\mathrm{OH})_{2}$ 沉淀对有机 质的吸附作用有关, 在 2 次 MAGIC 操作中 $\mathrm{Mg}(\mathrm{OH})_{2}$ 沉淀量基本不变, 故吸附的有机质含量变化不大, 减少 的部分可能是被 $\mathrm{NaOH}$ 清洗掉的碱溶性有机质. 由于 MAGIC 操作复杂, 且耗时长, 因此, 建议只采用 1 次 MAGIC.

在利用特征沉淀对样品进行纯化的步骤中, Blake 法与 McLaughlin 法分别采用了 APM + MAP 沉淀和 $\mathrm{CePO}_{4}$ 沉淀, 但是二者对有机质的去除效果有显著差异 $(P=0.007)$. 经 APM+MAP 沉淀处理后, 样品中 DOC 浓度减少至 $7.74 \pm 1.13 \mathrm{mg} / \mathrm{L}$, 约为提取液 DOC 浓度的 $12.70 \%$; 而 $\mathrm{CePO}_{4}$ 沉淀却使 DOC 浓度升高至提取液的 2 倍. Blake 法中 APM 和 MAP 沉淀分别选用 5\% $\mathrm{NH}_{4} \mathrm{NO}_{3}$ 和 $1: 20$ 氨水进行洗涤,2 种试剂可分别去除酸溶性 有机质和碱溶性有机质, 从而使 DOC 浓度下降. McLaughlin 法中 DOC 浓度升高的原因可能是在第一步中阳 离子交换树脂用量不足, 导致金属离子无法完全去除, 在 $\mathrm{CePO}_{4}$ 沉淀的过程中, 生成金属氢氧化物. 在洗涤 沉淀的过程中, 大量 $\mathrm{CH}_{3} \mathrm{COOK}$ 会吸附于沉淀, 且固液分离时有少量 $\mathrm{CH}_{3} \mathrm{COOK}$ 溶液仍滞留在离心管内, 导 致 DOC 浓度升高.

经过阴离子交换树脂处理后, Blake 法处理的样品中 DOC 浓度几乎没有变化, 表明在 DOC 浓度较低的 情况下, 阴离子树脂对有机质的去除效果不明显. 因此, 建议省略阴离子交换树脂处理. McLaughlin 法处理 的样品中 DOC 浓度减少至 $22.63 \pm 7.98 \mathrm{mg} / \mathrm{L}$, 约为提取液中 DOC 浓度的 $1 / 3$, 但仍明显高于 Blake 法的处理 
结果. $\mathrm{CH}_{3} \mathrm{COOH}$ 和 $\mathrm{PO}_{4}^{3-}$ 对阴离子交换树脂的相对选择性十分接近, 分别为 5.0 和 3.2(BIO-RAD 公司), 部分 $\mathrm{CH}_{3} \mathrm{COOH}$ 会随 $\mathrm{PO}_{4}^{3-}$ 一同发生吸附解吸而无法去除, 从而导致 DOC 浓度较高.
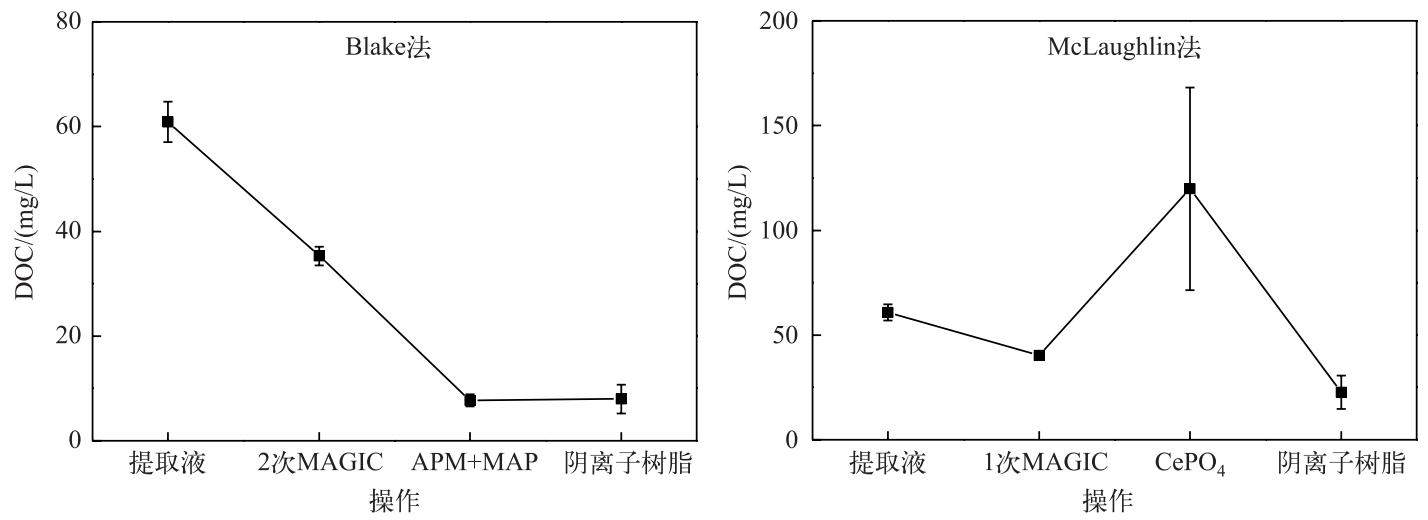

图 3 Blake 法和 McLaughlin 法对有机质去除效率对比

Fig.3 Comparison of organic matter removal efficiency between Blake and McLaughlin methods

2.2.2 不同方法的磷回收率比较 Blake 法和 McLaughlin 法对磷的总回收率接近, 但 Blake 法稳定性较好 (表 2). MAGIC 法几乎不造成磷损失, 且 1 次操作与 2 次操作结果没有显著性差异 $(P=0.126)$. $\mathrm{CePO}_{4}$ 沉淀与 $\mathrm{APM}+\mathrm{MAP}$ 沉淀的回收率基本一致,但 $\mathrm{CePO}_{4}$ 沉淀操作相对复杂, 因 $\mathrm{CePO}_{4}$ 沉淀受 $\mathrm{pH}$ 影响较大, McLaughlin 研究得出 $\mathrm{pH}=5.5$ 时效果最好 ${ }^{[12]}$. 通常, 沉积物提取液中含有大量的 $\mathrm{Fe} 、 \mathrm{Al} 、 \mathrm{Ca}$ 等金属离子, 在 $\mathrm{pH}$ 调节过程 中极易产生金属离子的氢氧化物沉淀, 从而干扰 $\mathrm{CePO}_{4}$ 沉淀的生成. 在本实验中,为了降低金属离子的干扰 作用, 在 MAGIC 操作前增加了阳离子树脂除去杂质金属离子, 以便更好地生成 $\mathrm{CePO}_{4}$. 阳离子树脂对回收 率的影响较小, 少量的 $\mathrm{PO}_{4}^{3-}$ 会残留在树脂表面,纯水多次冲洗即可提高回收率. 阴离子树脂对回收率的影响 较大, 按照阴离子树脂对抗衡离子的选择性顺序, $\mathrm{HSO}_{4}^{-} 、 \mathrm{NO}_{3}^{-}$等杂质离子会优先吸附到树脂上, 减少了 $\mathrm{PO}_{4}^{3-}$ 与树脂结合的机会, 未能吸附到阴离子树脂上的 $\mathrm{PO}_{4}^{3-}$ 将随废液损失掉; 此外, 阴离子树脂操作复杂, 耗时长, 且对树脂的用量要求较高, 树脂不足会增加磷损失的机率.

表 2 各步骤磷回收率比较

Tab.2 Overall phosphate throughput during purification

\begin{tabular}{|c|c|c|c|}
\hline \multicolumn{2}{|c|}{ Blake 法 } & \multicolumn{2}{|c|}{ McLaughlin 法 } \\
\hline 操作 & 回收率 & 操作 & 回收率 \\
\hline 2 次 MAGIC & $100.1 \% \sim 100.9 \%$ & AG50W-X8( 阳) & $97.0 \% \sim 100.4 \%$ \\
\hline APM+MAP 沉淀 & $84.1 \% \sim 86.1 \%$ & 1 次 MAGIC & $100.5 \% \sim 102.6 \%$ \\
\hline AG50W-X8(阳) & $90.2 \% \sim 97.7 \%$ & $\mathrm{CePO}_{4}$ 沉淀 & $82.6 \% \sim 89.1 \%$ \\
\hline AG1-X8(阴) & $90 \% \sim 95 \%$ & AG50W-X8( 阳) & $90.0 \% \sim 97.2 \%$ \\
\hline AG50W-X8(阳) & $95 \% \sim 98 \%$ & AG1-X8(阴) & $77.0 \% \sim 101.4 \%$ \\
\hline 合计 & $64.8 \% \sim 78.3 \%$ & 合计 & $55.5 \% \sim 86.6 \%$ \\
\hline
\end{tabular}

\section{3 两种经典方法的纯化效果比较}

按照 Blake 法, 将纯化后的溶液蒸发浓缩, 加人银氨溶液后加热使氨水挥发析出 $\mathrm{Ag}_{3} \mathrm{PO}_{4}$, 亮黄色的结晶 浮于溶液表面. 按照 McLaughlin 法, 调节 $\mathrm{pH}$ 至 7 8, 加人 $\mathrm{AgNO}_{3}$ 溶液, 迅速生成黄白色的 $\mathrm{Ag}_{3} \mathrm{PO}_{4}$ 沉淀, 沉淀 随时间慢慢变暗.

为了比较 $\mathrm{Ag}_{3} \mathrm{PO}_{4}$ 纯度, 分别对 2 种方法得到的 $\mathrm{Ag}_{3} \mathrm{PO}_{4}$ 进行 $\mathrm{C} 、 \mathrm{~N}$ 含量测定 (图 4). 其中 1 7 和 8 13 分 别代表用 Blake 法和 McLaughlin 法纯化样品 3 得到的 $\mathrm{Ag}_{3} \mathrm{PO}_{4}$ 平行样. 经 Blake 法纯化获得的 $\mathrm{Ag}_{3} \mathrm{PO}_{4}$ 的 $\mathrm{C} 、 \mathrm{~N}$ 
含量分别为 $0.622 \% \pm 0.081 \%$ 和 $0.032 \% \pm 0.006 \%$; 经 McLaughlin 法纯化获得的 $\mathrm{Ag}_{3} \mathrm{PO}_{4}$ 的 $\mathrm{C} 、 \mathrm{~N}$ 含量分别为 $3.37 \% \pm 0.82 \%$ 和 $0.55 \% \pm 0.21 \%$, 分别是 Blake 法的 5.4 和 17.2 倍, 说明 Blake 法的纯化效果更好, 重现性高, 效果稳定. 这与 Tamburini 等 ${ }^{[15]}$ 的研究结果一致. Tamburini 等在分析不同有机质含量的土壤样品的 $\delta^{18} \mathrm{O}_{\mathrm{P}}$ 时 发现, 通过 McLaughlin 法纯化获得的 $\mathrm{Ag}_{3} \mathrm{PO}_{4}$ 中含有有机质, 且对后续的氧同位素测定有干扰; 慢沉淀可以 减少有机质的混人和对 $\delta^{18} \mathrm{O}_{\mathrm{P}}$ 测定的影响 ${ }^{[15]}$.

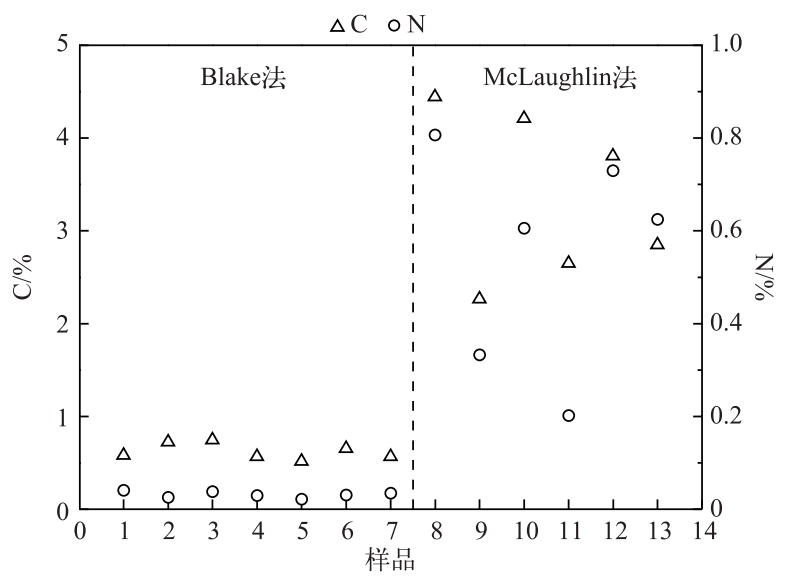

图 4 经 Blake 法和 McLaughlin 法获得的 $\mathrm{Ag}_{3} \mathrm{PO}_{4}$ 的 $\mathrm{C} 、 \mathrm{~N}$ 含量对比

Fig.4 Comparison of $\mathrm{C}$ and $\mathrm{N}$ contents of $\mathrm{Ag}_{3} \mathrm{PO}_{4}$ through Blake and McLaughlin methods

\section{3 结论}

1 ) $\mathrm{NaClO}$ 处理可以保证较高的无机磷提取效率, 同时能有效地减少无机磷提取液中有机质含量, 且对 不同形态磷的破坏较小; $\mathrm{H}_{2} \mathrm{O}_{2}$ 对有机质的去除效果不稳定, 处理后沉积物的磷形态之间发生转化. 因此, $\mathrm{NaClO}$ 法更适用于湖泊沉积物样品的预处理.

2) Blake 法对有机质的去除率高于 McLaughlin 法;2 种方法的磷回收率差异不大,但 Blake 法稳定性较 好. Blake 法和 McLaughlin 法得到的 $\mathrm{Ag}_{3} \mathrm{PO}_{4}$ 中 $\mathrm{C} 、 \mathrm{~N}$ 含量的对比分析表明, Blake 法的纯化效果更好且更 稳定.

3 ) 结合 NaClO 法与 Blake 法的优点, 建立了一种适合湖泊沉积物的磷酸盐氧同位素前处理方法 (图 5 ). 首先用 $2.5 \% \mathrm{NaClO}$ 对沉积物样品进行除碳预处理, 然后对磷酸盐提取液依次通过氢氧化镁共沉淀 (MAGIC)、APM+MAP 沉淀、阳离子交换树脂处理,最后生成 $\mathrm{Ag}_{3} \mathrm{PO}_{4}$.

\section{4 参考文献}

[ 1 ] Li Bo ed. Ecology. Beijing: Higher Education Press, 2000: 251-252. [李博. 生态学. 北京: 高等教育出版社, 2000: 251-252.]

[ 2 ] Fan Chengxin. Physiochemical characteristics of sedimets in Gehu Lake and simulation of its phosphorus release. $J$ Lake Sci, 1995, 7(4) : 341-349. DOI: 10.18307/1995.0408. [范成新. 滆湖沉积物理化特征及磷释放模拟. 湖泊科学, $1995,7(4): 341-349$.

[ 3 ] Song Yuanyuan, Feng Muhua, Su Zhengguang et al.Vertical distribution of chemical speciation of phosphorus in sediments from different sources of Fuxian Lake. Acta Scientiae Circumstantiae, 2013, 33(9): 2579-2589. [ 宋媛媛, 冯慕华, 苏争 光等. 抚仙湖不同来源沉积物磷形态垂向分布特征. 环境科学学报,2013, 33(9) : 2579-2589.]

[ 4 ] Davies CL, Surridge BW, Gooddy DC. Phosphate oxygen isotopes within aquatic ecosystems: Global data synthesis and future research priorities. Science of the Total Environment, 2014, 496: 563-575.

[ 5 ] Liang Y, Blake R. Oxygen isotope signature of Pi regeneration from organic compounds by phosphomonoesterases and pho- 


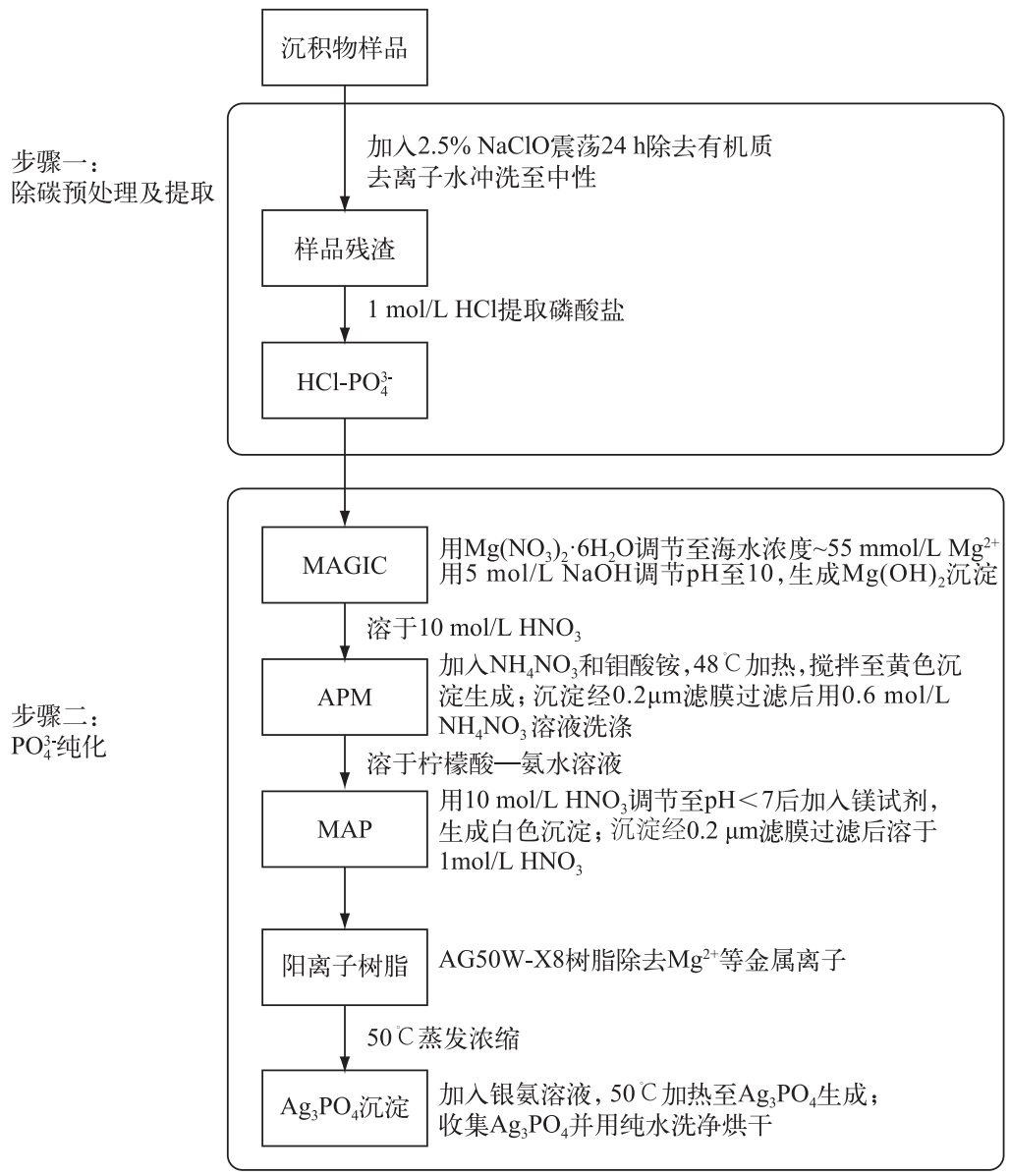

图 5 湖泊沉积物磷酸盐氧同位素前处理流程图

Fig.5 Procedure for pretreatment of oxygen isotope composition of phosphate in lake sediments tooxidation. Geochimica et Cosmochimica Acta, 2006, 70(15) : 3957-3969.

[ 6 ] Mclaughlin K, Paytan A, Kendall C et al.Phosphate oxygen isotopes as a tracer for sources and cycling of phosphate in San Francisco Bay. Journal of Geophysical Research, 2006, 111, G03003.

[ 7 ] Jaisi DP, Blake RE. Tracing sources and cycling of phosphorus in Peru Margin sediments using oxygen isotopes in authigenic and detrital phosphates. Geochimica et Cosmochimica Acta, 2010, 74(11) : 3199-3212.

[ 8 ] Goldhammer T, Brunner B, Bernasconi SM et al.Phosphate oxygen isotopes: Insights into sedimentary phosphorus cycling from the Benguela upwelling system. Geochimica et Cosmochimica Acta, 2011, 75(13) : 3741-3756.

[ 9 ] Stephan E. Oxygen isotope analysis of animal bone phosphate: Method refinement, influence of consolidants, and reconstruction of palaeotemperatures for holocene sites. Journal of Archaeological Science, 2000, 27 : 523-535.

[10] O'Neil JR, Roe LJ, Reinhard E et al. A rapid and precise method of oxygen isotope analysis of biogenic phosphate. Israel Journal of Earth Sciences, 1994, 43(3/4) : 203-212.

[11] Blake RE, Chang SJ, Lepland A. Phosphate oxygen isotopic evidence for a temperate and biologically active Archaean ocean. Nature, 2010, 464(7291): 1029-1032.

[12] Mclaughlin K, Silva S, Kendall C et al. A precise method for the analysis of $\delta^{18} \mathrm{O}$ of dissolved inorganic phosphate in seawater. Limnology and Oceanography: Methods, 2004, 2(7) : 202-212.

[13] Lu Yangyang, Zheng Zhenzhen, Yin Xijie et al. The measurement of oxygen isotope composition of dissolved inorganic phosphate in seawater. Acta Geoscientica Sinica, 2012, 33(6) : 961-966. [卢阳阳, 郑珍珍, 尹希杰等. 海水溶解磷酸盐 
氧同位素组成的测定. 地球学报, 2012, 33(6): 961-966.]

[14] Mclaughlin K, Cade-Menun BJ, Paytan A. The oxygen isotopic composition of phosphate in Elkhorn Slough, California: A tracer for phosphate sources. Estuarine, Coastal and Shelf Science, 2006, 70(3): 499-506.

[15] Tamburini F, Bernasconi S, Angert A et al. A method for the analysis of the $\delta 180$ of inorganic phosphate extracted from soils with HCl. European Journal of Soil Science, 2010, 61(6) : 1025-1032.

[16] Egemose S, De Vicente I, Reitzel K et al. Changed cycling of P, N, Si, and DOC in Danish Lake Nordborg after aluminum treatment. Canadian Journal of Fisheries and Aquatic Sciences, 2011, 68(5) : 842-856.

[17] Zhang JZ, Guo L, Fischer CJ. Abundance and chemical speciation of phosphorus in sediments of the Mackenzie River Delta, the Chukchi Sea and the Bering Sea: Importance of detrital apatite. Aquatic Geochemistry, 2010, 16(3) : 353-371.

[18] Editorial Board of Water and Wastewater Monitoring and Analysis Methods, Ministry of Environmental Protection of the People's Republic of China ed. Water and wastewater monitoring and analysis methods: 4th edition. Beijing: China Environmental Science Press, 2002. [ 国家环境保护总局《水和废水监测分析方法》编委会. 水和废水监测分析方法:第 4 版. 北京: 中国环境科学出版社, 2002.]

[19] Blake RE, Chang SJ, Lepland A. Phosphate oxygen isotopic evidence for a temperate and biologically active Archaean ocean. Nature, 2010, 464(7291) : 1029-1032.

[20] Lavkulich LM, Wiens JH. Comparison of organic matter destruction by hydrogen peroxide and sodium hypochlorite and its effects on selected mineral constituents. Soil Science Society of America Journal, 1970, 34: 755-758.

[21] Mikutta R, Kleber M, Kaiser K et al. Review : Organic matter removal from soil using hydrogen peroxide, sodium hypochlorite, and disodium peroxodisulfate. Soil Science Society of America Journal, 2005, 69:121-135.

[22] Siregar A, Kleber M, Mikutta R et al.Sodium hypochlorite oxidation reduces soil organic matter concentrations without affecting inorganic soil constituents. European Journal of Soil Science, 2005, 56(4) : 481-490.

[23] Anderson J. An improved pretreatment for mineralogical analysis of samples containing organic matter. Clays and Clay Minerals, $1963, \mathbf{1 0}(3)$ : 380-388.

[24] Jimenez MCS, Dominguez AP, Silverio JMC. Reaction kinetics of humic acid with sodium hypochlorite. Water Research, 1993, 27(5): 815-820.

[25] Li CW, Benjamin MM, Korshin GV. Use of UV spectroscopy to characterize the reaction between NOM and free chlorine. Environmental Science \& Technology, 2000, 34(12) : 2570-2575.

[26] Pomes ML, Larive CK, Thurman EM et al. Sources and haloacetic acid/trihalomethane formation potentials of aquatic humic substances in the Wakarusa River and Clinton Lake near Lawrence, Kansas. Environmental Science \& Technology, $2000,34(20):$ 4278-4286.

[27] Strukul G. Catalytic oxidations with hydrogen peroxide as oxidant. Springer Science \& Business Media. Dordrecht: Kluwer Academic Publisher, 1992: 97-98.

[28] Wang GS, Liao CH, Wu FJ. Photodegradation of humic acids in the presence of hydrogen peroxide. Chemosphere, 2001, 42(4): 379-387.

[29] Chakrabartty S, Kretschmer H, Cherwonka S. Hypohalite oxidation of humic acids. Soil Science, 1974, 117( 6) : 318-322.

[30 ] Yin Ran, Wang Fushun, Mei Hangyuan et al.Distribution of phosphorus forms in the sediments of cascade reservoirs with different trophic states in Wujiang catchment. Chinese Journal of Ecology, 2010, 29(1) : 91-97. [尹然, 汪福顺, 梅航远 等. 乌江流域不同营养水平的梯级水库沉积物中磷形态特征. 生态学杂志, 2010, 29(1): 91-97.]

[31 ] Pan B, Wu J, Pan B et al. Development of polymer-based nanosized hydrated ferric oxides (HFOs) for enhanced phosphate removal from waste effluents. Water Research, 2009, 43(17) : 4421-4429. 